\title{
Effective Sensing Devices for Online Monitoring of Urea and Creatinine in Human Urine
}

\author{
Sumonmarn Chaneam ${ }^{1,2 *}$, Kampanart Kaewyai ${ }^{2,3}$, Rasamee Chaisuksant ${ }^{1}$ and Duangjai Nacapricha ${ }^{2,3}$ \\ ${ }^{1}$ Department of Chemistry, Faculty of Science, Silpakorn University, \\ Nakhon Pathom, 73000, Thailand \\ 2 Flow Innovation-Research for Science and Technology Laboratories (FIRST Labs.) \\ ${ }^{3}$ Department of Chemistry and Center of Excellence for Innovation in Chemistry, Faculty of Science, \\ Mahidol University, Rama 6 Road, Bangkok 10400, Thailand \\ *schaneam@gmail.com
}

\begin{abstract}
:
In this work, effective and low cost analytical devices were constructed using the concepts of contactless conductivity detector and colorimetric detector. Then, the developed devices were integrated with an automatic flow injection analysis (FIA) for online monitoring of urea and creatinine in human urine samples. The simultaneous determination was operated by injection of urine sample into a carrier stream and flow through a urease column. Urea was hydrolyzed by urease to produce $\mathrm{NH}_{3}$. Using a gas diffusion unit, the generated $\mathrm{NH}_{3}$ in a carrier stream was separated and trapped into an acceptor stream of deionized water as $\mathrm{NH}_{4}{ }^{+}$form. Changing of conductivity related to the concentration of urea in sample was detected with the in-house contacless conductivity detector. The same sample zone of urine in the donor stream was then subsequently flowed to mix with alkaline picrate for creatinine determination. The colored change of solution after reaction was determined by the in-house colorimetric detector. The proposed system was successfully applied to analysis of urea and creatinine in urine samples. It was found that the method developed here can give similar results compared to those obtained from the reference methods.
\end{abstract}

Key words: Flow injection analysis, Contactless conductivity detector, Colorimetric detector, Urea, Creatinine

\section{Introduction}

Urine is produced in the kidneys by a process called urination. It composes of mainly water and certain other water soluble organic and inorganic compounds. The main organic compounds present in the urine include urea and creatinine. Urea and creatinine are the waste formed as a result of metabolic reaction in the body. Accumulation of these waste products take place in the body due to renal failure. Since, urea is synthesized as a result of amino acid metabolism, the amount of urea in the urine reflects the amount of protein degradation. The normal range of urea and creatinine in a hundred milliliter of urine are about $1500 \mathrm{mg}$ and $100 \mathrm{mg}$, respectively. Despite the automatic machine have been already used for monitoring the content of urea and creatinine, it is only found in the central hospital. Therefore, there is a need to develop a simple and economical method for analysis of both urea and creatinine. This work presents the construction of a contactless conductivity detector and colorimetric detector and use them in the flow injection analysis (FIA) system for simultaneous analysis. The developed method was then applied to urea and creatinine analysis in human urine samples.

\section{Experiment}

In order to construct the calibration curve of urea in parallel with creatinine, mixed standard solutions of urea and creatinine were used. For urine analysis, the urine samples were collected and diluted with deionized water. Schematic diagram of the proposed system was illustrated in Fig. 1. A peristaltic pump (ISMATEC, Switzerland) was used to promote the fluid propulsion with a flow rate of $1.0 \mathrm{~mL} \mathrm{~min} \mathrm{~m}^{-1}$. The standard or sample was injected via a 6-port injection valve (V-540, Upchurch Scientific, Germany) and was then carried through a urease column [1] by a donor stream of buffer at $\mathrm{pH}$ 8.80. Urea was hydrolyzed by urease to produce $\mathrm{NH}_{3}$ gas. Using a gas diffusion unit (GD), the generated $\mathrm{NH}_{3}$ gas in the donor stream was diffused through the PTFE hydrophobic membrane (47 $\mathrm{mm}$ i.d. with pore size $0.45 \mu \mathrm{m}$, Sartorius, Germany) and trapped into an acceptor stream of deionized water. Changing of conductivity of the acceptor stream was detected with the lab-made contactless conductivity detector [2]. At the same time, the same sample zone in the donor stream was then subsequently flowed to mix with the Jaffe reagent, which is alkaline picrate, for creatinine determination. The red-orange product zone was detected by an in-house LED-based colorimetric detector [3]. The two data sets of conductivity and color measurements were recoded and stored on a computer with in-house software written using Lab VIEW 8.2 ${ }^{\mathrm{TM}}$. 


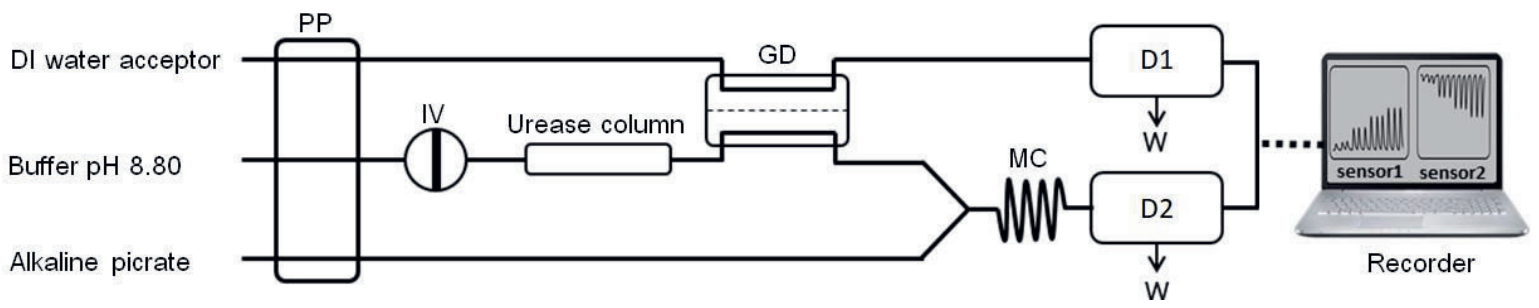

Fig.1. Schematic diagram of the proposed FIA system for simultaneous determination of urea and creatinine. PP, peristaltic pump; IV, injection valve; GD, gas diffusion unit; $M C$, mixing coil (i.d. $1 \mathrm{~mm}, 120 \mathrm{~mm}$ long); D1, contactless conductivity detector; $D 2$, colorimetric detector; $W$, Waste.

\section{Results and Discussion}

Signal profiles and calibration curves obtained from mixture of standard urea and creatinine were shown in Fig. 2. It should be noted that the linear calibration curve from both analytes were obtained simultaneously. The potentiometric analytical signals picked up from our sensing devices were proportional to the sample conductivity (2a) as well as color intensity (2b) and thus correlated to the analyte concentration. At the optimum conditions, linear ranges of $30-240 \mathrm{mg} \mathrm{L}^{-1}$ and $10-500 \mathrm{mg} \mathrm{L}^{-1}$ were obtained for urea and creatinine, respectively. High throughput of 30 samples $\mathrm{h}^{-1}$ was achieved.
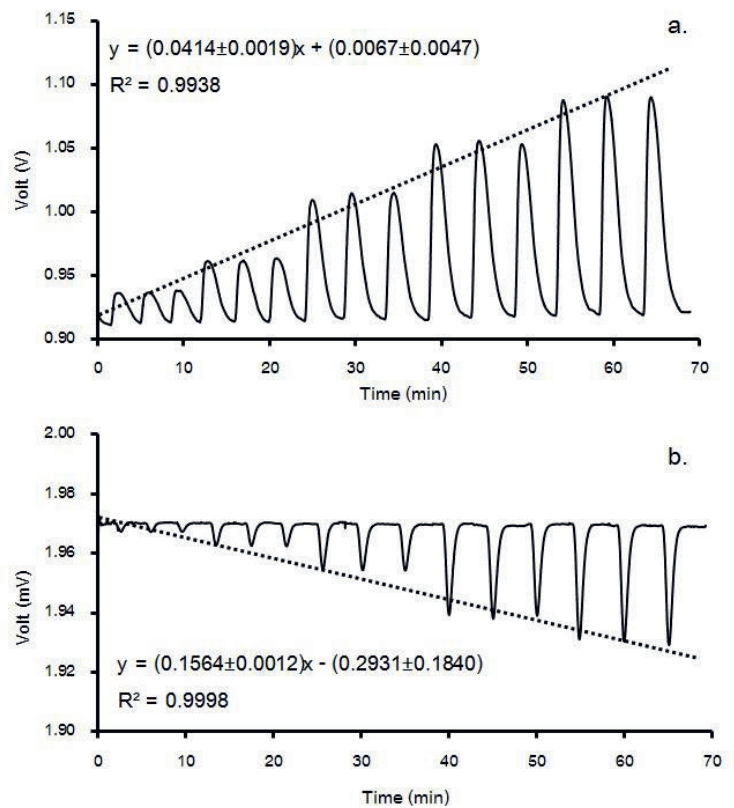

Fig. 2. Signal profile of urea (a) and creatinine (b) together with the calibration curves obtained from the FIA system operated at flow rate of $1 \mathrm{~mL} \mathrm{~min}^{-1}$ and sample volume of $100 \mu \mathrm{L}$.

This developed method has been applied to determine urea and creatinine in urine samples. The recovery was between $90-118 \%$, indicated that our system could be effectively used for simultaneous quantitative analysis of urea and creatinine in urine. Finally, the results of the analysis by our method were validated with those from reference methods as shown in Fig. 3. It has been clearly seen that our method statistically gave good agreement with the reference methods.
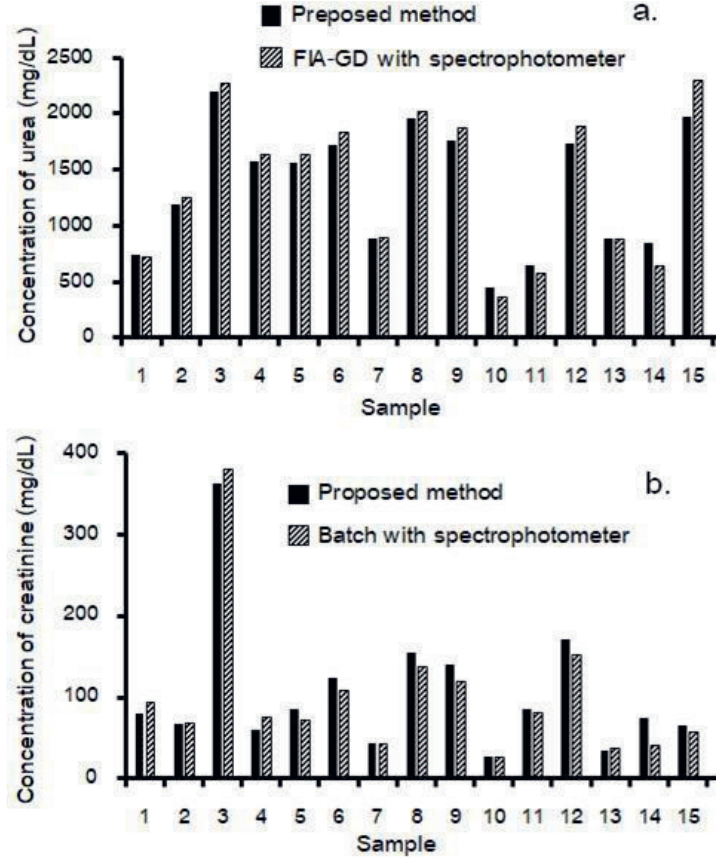

Fig. 3. Analysis results of urea (a) and creatinine (b) from our proposed method and reference methods.

\section{Conclusion}

The proposed system provides rapid screening feature and economic technique for simultaneous determination of the two biomarkers of renal function, urea and creatinine. We successfully demonstrated the use of our method for urine analysis which is comparable to the reference methods. We hope that our method would be developed to an alternative low cost method for application in local hospital.

\section{Acknowledgments}

The authors would like to thank the Institute for the Promotion of Teaching Science and Technology, Thailand, for a DPST Research Grant (017/2557). Thailand Research Fund (MRG6080182) is gratefully acknowledged.

\section{References}

[1] W. Limbut, P. Thavarungkul, P. Kanatharana, P. Asawatreratanakul, C. Limsakul. B. Wongkittisuksa, Biosens Bioelectron 19 (2004) 813.

[2] L. H. Braz, T. D. Ito, A. J. Fracassi da Silva, L. C. do Lago, J. J. Pedrotti, Electroanalysis 23 (2011) 2594.

[3] Ł. Tymecki, J. Korszun, K. Strzelak, R. Koncki, Anal. Chim. Acta 787 (2013) 118. 